PROCEEDINGS OF THE

AMERICAN MATHEMATICAL SOCIETY

Volume 137, Number 6, June 2009, Pages 2005-2014

S 0002-9939(09)09819-0

Article electronically published on January 16, 2009

\title{
EXISTENCE AND UNIQUENESS OF TRONQUÉE SOLUTIONS OF THE FOURTH-ORDER JIMBO-MIWA SECOND PAINLEVÉ EQUATION
}

\author{
NALINI JOSHI AND TEGAN MORRISON \\ (Communicated by Peter A. Clarkson)
}

\begin{abstract}
We consider the asymptotic limit as the independent variable approaches infinity, of the fourth-order second Painlevé equation obtained from a hierarchy based on the Jimbo-Miwa Lax pair. We prove that there exist two families of algebraic formal power series solutions and that there exist true solutions with these behaviours in sectors $\sigma$ of the complex plane. Given $\sigma$ we also prove that there exists a wider sector $\Sigma \supset \sigma$ in which there exists a unique solution in each family. These provide the analogue of Boutroux's tri-tronquée solutions for the classical second Painlevé equation. Surprisingly, they also extend beyond the tri-tronquée solutions in the sense that we find penta-, hepta-, ennea-, and hendeca-tronquée solutions.
\end{abstract}

\section{INTRODUCTION}

The asymptotics of the classical second Painlevé equation

$$
\mathrm{P}_{\mathrm{II}}: \quad y_{x x}=2 y^{3}+x y+\alpha, \quad|x| \rightarrow \infty, \quad \alpha=\text { constant, }
$$

have been widely studied. While the generic solution is known to possess poles in every neighbourhood of infinity in the complex plane, there are special families of solutions that do not have poles in every subsector of such a neighbourhood. Boutroux [1, 2] proved the existence of two one-parameter families of solutions of $\mathrm{P}_{\mathrm{II}}$ that he called tronquée, and of six unique solutions called tri-tronquée. The solutions are asymptotic to

$$
y(x)=\left(\frac{-x}{2}\right)^{1 / 2}\left(1+\mathcal{O}\left(x^{-3 / 2(1-\epsilon)}\right)\right) \text { or } y(x)=\frac{-\alpha}{x}\left(1+\mathcal{O}\left(x^{-3 / 2(1-\epsilon)}\right)\right),
$$

$\epsilon>0$, in particular annular sectors of the plane as $|x| \rightarrow \infty$. There exists $x_{0} \neq 0$, such that the tronquée solutions are pole free in the sectors

$$
\Omega_{k}=\left\{x \in \mathbb{C}|| x|>| x_{0} \mid,(k-1) \pi / 3<\arg (x)<(k+1) \pi / 3\right\}, \quad k=0, \ldots, 5 .
$$

Boutroux pointed out that each such sector is bisected by a ray given by $\arg x=$ $k \pi / 3$, and that no poles are present beyond a circle of some finite radius along such a ray. Similarly, the tri-tronquée solutions are pole free in extended sectors

Received by the editors October 16, 2007, and, in revised form, April 9, 2008.

2000 Mathematics Subject Classification. Primary 33E17, 34M55.

The authors gratefully acknowledge the support of the Australian Research Council through Discovery Grant DP0559019 and an Australian Postgraduate Award.

(C)2009 American Mathematical Society Reverts to public domain 28 years from publication 
$\Omega_{k} \cup \Omega_{k+2}$ within which lie three bisectors of the original sectors. Such asymptotic results lead to complex analytic information about the solutions. In the case of the Painlevé equations this is particularly useful because, in generic cases, the solutions are highly transcendental functions whose explicit analytic properties have been difficult to deduce.

$\mathrm{P}_{\mathrm{II}}$ is one of six classical Painlevé equations which are regarded as completely integrable because they can be solved through an associated system of linear equations. $\mathrm{P}_{\mathrm{II}}$ has two well-known linear problems given by Flaschka and Newell in [4, and Jimbo and Miwa in [9. $\mathrm{P}_{\mathrm{II}}$ is also the first member of two infinite hierarchies of ODEs, which arise as symmetry reductions of two different PDE hierarchies: the modified KdV hierarchy, and a dispersive water wave hierarchy. The reduction of these equations and their associated linear problems are given explicitly in [1] and [5], respectively. For $\mathrm{P}_{\mathrm{II}}$, it is important to recognise that the two reductions lead to each of the two different linear problems given by Flaschka and Newell, and Jimbo and Miwa (see [6]), respectively. We therefore distinguish the two hierarchies by those names, and denote them $\mathrm{FN} \mathrm{P}_{\mathrm{II}}^{(n)}$ and $\mathrm{JM} \mathrm{P}_{\mathrm{II}}^{(n)}$, respectively.

In this paper, we concentrate on the Jimbo-Miwa hierarchy and show that tronquée-type solutions also exist for this hierarchy. To illustrate the results, we concentrate on the second member of the Jimbo-Miwa hierarchy,

$$
\text { JM P } \mathrm{P}_{\mathrm{II}}^{(2)}: \quad \begin{aligned}
u_{x x}-3 u u_{x}+u^{3}+6 u v+4 x g_{3} & =0, \\
v_{x x}+3 u v_{x}+3 v^{2}+3 u^{2} v & =4 \alpha_{2} .
\end{aligned}
$$

For each index $n \geq 1$, JM $\mathrm{P}_{\mathrm{II}}^{(n)}$ is defined by two $n$ th-order ODEs,

$$
\mathrm{JM} \mathrm{P} \mathrm{II}_{\mathrm{II}}^{(n)}: \quad \mathcal{A}_{n}+2^{n} g_{n+1}\left(\begin{array}{c}
x \\
0
\end{array}\right)=2^{n}\left(\begin{array}{c}
0 \\
\alpha_{n}
\end{array}\right),
$$

where $g_{n+1} \neq 0$ and $\alpha_{n}$ are constants, and $\mathcal{A}_{n}$ is defined recursively as

$$
\mathcal{A}_{n}=\mathcal{R} \mathcal{A}_{n-1}, \quad \mathcal{A}_{0}=\left(\begin{array}{l}
u \\
v
\end{array}\right), \quad \mathcal{R}=\left(\begin{array}{cc}
u-\partial_{x} & 2 \\
2 v-\partial_{x}^{-1} v_{x} & u-\partial_{x}^{-1} u_{x}+\partial_{x}
\end{array}\right) .
$$

Asymptotics of the hierarchy JM $\mathrm{P}_{\mathrm{II}}^{(n)}$ will be studied in a subsequent work.

The first member of the hierarchy,

$$
\begin{aligned}
\mathrm{JM} \mathrm{P}_{\mathrm{II}}^{(1)}: \quad-u_{x}+u^{2}+2 v+2 x g_{2} & =0, \\
v_{x}+2 u v & =2 \alpha_{1},
\end{aligned}
$$

is equivalent to $\mathrm{P}_{\mathrm{II}}$ in $u$, and $\mathrm{P}_{34}$ (of [7]) in $v$. Similarly, the system $\mathrm{JM} \mathrm{P}_{\mathrm{II}}^{(2)}$ (1.1) is equivalent to a fourth-order scalar equation in $u$,

$$
\begin{aligned}
u_{x x x x} & =\frac{2 u_{x} u_{x x x}}{u}+\frac{3 u_{x x}^{2}}{2 u}-\frac{2 u_{x}^{2}}{u_{x x}} u+5 u^{2} u_{x x}+\frac{8 x g_{3} u_{x x}}{u}+\frac{5}{2} u u_{x}^{2} \\
& -\frac{8 x g_{3} u_{x}^{2}}{u^{2}}+\frac{8 g_{3} u_{x}}{u}-\frac{5}{2} u^{5}-8 x g_{3} u^{2}-12 u\left(2 \alpha_{2}+g_{3}\right)+\frac{8 x^{2} g_{3}^{2}}{u} .
\end{aligned}
$$

It is interesting to note that this equation also appears in other studies. In particular, it appears in a study of caustic-type limits of PDEs [13, and in [14, where higher-order equations are constructed by requiring that the resulting equations have hyper-elliptic functions as asymptotic behaviours. Equation (1.2) appears to 
be quite different from the other fourth-order analogue of $\mathrm{P}_{\mathrm{II}}$, or the second member of the Flaschka-Newell hierarchy,

$$
\mathrm{FN} \mathrm{P}_{\mathrm{II}}^{(2)}: \quad y_{x x x x}-10 y^{2} y_{x x}-10 y y_{x}^{2}+6 y^{5}=x y+\alpha_{2} .
$$

An important open question is whether these two equations are related through a transformation of variables, or even whether they admit the same types of qualitative asymptotic behaviours in the limit as $|x| \rightarrow \infty$.

Tronquée-type solutions of equation (1.3) were considered by Joshi and Mazzocco [10. Here, we ask whether tronquée-type solutions of equation (1.1) exist. Painlevé equations, and hierarchies, appear in the study of string equations of certain matrix models [15], and in the study of gradient catastrophe in wave equations, including the focusing non-linear Schrödinger equation [3]. Remarkably, the physically important solutions in these settings turn out to be those that are pole free, or tronquée, in certain sectors.

In this paper, we show that there are two families of pole-free solutions for JM $\mathrm{P}_{\mathrm{II}}^{(2)}$. We find a wider variety of tronquée-type solutions than have been previously considered. In particular, in addition to tronquée and tri-tronquée solutions, there exist what we call penta-, hepta-, ennea-, and hendeca-tronquée solutions. We give explicit details on the asymptotic expansion of the solutions, and the size and orientation of the sectors of validity of the asymptotic description of the solutions.

The plan of the paper is as follows. We construct formal solutions in $\S 2$ and prove their existence in $\S 3$. In $\S 4$, we prove that a subset of these solutions is unique and suggest a naming convention of these new solutions in $\S 5$. We conclude with a discussion in $\S 6$.

\section{Formal SOLUtions}

Proposition 2.1. In the limit as $|x| \rightarrow \infty$, the system of equations JM $P_{\mathrm{II}}^{(2)}$ (1.1) has two families of formal solutions:

$$
\begin{aligned}
& u_{A, j, f}=\omega_{j}\left(-4 g_{3} x\right)^{1 / 3} \sum_{i=0}^{\infty} \frac{a_{i}}{\left(x^{1 / 3}\right)^{4 i}}, v_{A, j, f}=\frac{4 \alpha_{2}}{3 \omega_{j}^{2}\left(-4 g_{3} x\right)^{2 / 3}} \sum_{i=0}^{\infty} \frac{b_{i}}{\left(x^{1 / 3}\right)^{4 i}} \\
& u_{B, j, f}=\omega_{j}\left(\frac{4 g_{3} x}{5}\right)^{1 / 3} \sum_{i=0}^{\infty} \frac{d_{i}}{\left(x^{1 / 3}\right)^{4 i}}, v_{B, j, f}=-\omega_{j}^{2}\left(\frac{4 g_{3} x}{5}\right)^{2 / 3} \sum_{i=0}^{\infty} \frac{e_{i}}{\left(x^{1 / 3}\right)^{4 i}},
\end{aligned}
$$

where $w_{j}^{3}=1, j=1,2,3, a_{0}=b_{0}=d_{0}=e_{0}=1$, and $a_{i}, b_{i}, d_{i}, e_{i}$, are determined by substitution.

Proof. Substitute each series into (1.1).

For the remainder of the paper we choose $g_{3}$ to be real and positive, and define $c_{0}^{3}=-4 g_{3}$ and $c_{1}^{3}=4 g_{3} / 5$.

Remark 2.1. The solutions in Proposition 2.1 are readily constructed by making use of the Boutroux-transformation of variables

$$
u(x)=x^{1 / 3} U(z), \quad z=\frac{3}{4} x^{4 / 3}, \quad v(x)=x^{2 / 3} V(z),
$$

in the system (1.1). This change of variables is chosen such that there is a maximum number of dominant terms in the transformed equations (see [12]). Written in 
Boutroux coordinates,

$$
\begin{aligned}
U_{z z}-3 U U_{z}+U^{3}+6 U V+4 g_{3} & =\frac{3}{4 z}\left(U^{2}-U_{z}\right)+\frac{U}{8 z^{2}}, \\
V_{z z}+3 U V_{z}+3 V^{2}+3 U^{2} V & =\frac{3 \alpha_{2}}{z}-\frac{1}{4 z}\left(6 U V+5 V_{z}\right)+\frac{V}{8 z^{2}},
\end{aligned}
$$

the equations reveal which terms are dominant in the limit as $|z| \rightarrow \infty$. In this case two algebraic leading-order balances are possible:

Case A: $\quad(U, V)=(\mathcal{O}(1), \mathcal{O}(1 / z)), \quad$ Case B: $\quad(U, V)=(\mathcal{O}(1), \mathcal{O}(1))$.

Formal solutions of (2.4) corresponding to these balances are of the form

$$
\begin{array}{ll}
\text { CAse A: } \quad U(z)=c_{0} \sum_{i=0}^{\infty} u_{i} z^{-i}, & V(z)=\frac{\alpha_{2}}{c_{0}^{2} z} \sum_{i=0}^{\infty} v_{i} z^{-i}, \\
\text { CASe B: } \quad U(z)=c_{1} \sum_{i=0}^{\infty} u_{i} z^{-i}, & V(z)=-c_{1}^{2} \sum_{i=0}^{\infty} v_{i} z^{-i},
\end{array}
$$

where $u_{0}=v_{0}=1$ and $u_{i}, v_{i}$, are determined by substitution in each case. Returning to the original coordinates via (2.3), these formal solutions are written as in Proposition 2.1, where the subscripts A and B match the cases defined above.

\section{Existence of true solutions}

\section{Proposition 3.1.}

(1) There exist two-parameter solutions of $J M P_{\mathrm{II}}^{(2)}$ whose asymptotic behaviour as $|x| \rightarrow \infty$ is given by (2.1) or (2.2) in the respective sectors:

$$
\begin{aligned}
\sigma_{A, k, \beta} & =\left\{x \in \mathbb{C}|| x|>| x_{0} \mid, \frac{k \pi}{4}<\arg \left(x-x_{0}\right)<\frac{(k+\beta) \pi}{4}\right\}, \\
\sigma_{B, k} & =\left\{x \in \mathbb{C}|| x|>| x_{0} \mid, \frac{(2 k+1) \pi}{8}<\arg \left(x-x_{0}\right)<\frac{(2 k+7) \pi}{8}\right\},
\end{aligned}
$$

where $\beta=1$ or $2, k=0, \ldots, 7$, and $x_{0} \neq 0$.

(2) There exist one-parameter solutions of JM $P_{\mathrm{II}}^{(2)}$ whose asymptotic behaviour as $|x| \rightarrow \infty$ is given by (2.1) in the sectors $\sigma_{A, k, 3}$.

Remark 3.1. While there appear to be no visible free parameters in the asymptotic expansions (2.1) and (2.2), solutions with these behaviours have parameters hidden beyond all orders as coefficients of exponentially small terms.

Proof of Proposition 3.1. We prove the result in the transformed $z$ coordinates, and then apply (2.3) to obtain the required results in terms of $x$. To prove the existence of actual solutions with behaviour (2.5) or (2.6), we employ Wasow's existence theorem ([16], Theorem 12.1). The hypotheses of this theorem require that the system of equations under consideration is formally satisfied by an asymptotic expansion of the form $\sum_{i=1}^{\infty} y_{i} z^{-i}$, for some constants $y_{i}$, where we note that the expansion begins with the index $i=1$ and not $i=0$. We therefore make the following change of variables. 
CASE A: Let $(U, V)$ be a solution of (2.4) which is asymptotic to (2.5), and define $\widetilde{U}=U-c_{0}$ and $\widetilde{V}=V$. Then, $(\widetilde{U}, \widetilde{V})$ solves the system

$$
\begin{aligned}
\widetilde{U}_{z z}-3 c_{0} \widetilde{U}_{z}+3 c_{0}^{2} \widetilde{U}+6 c_{0} \widetilde{V}+\left(3 \widetilde{U} \widetilde{U}_{z}+6 \widetilde{U} \widetilde{V}+\widetilde{U}^{3}\right) & =\mathcal{O}(1 / z), \\
\widetilde{V}_{z z}+3 c_{0} \widetilde{V}_{z}+3 c_{0}^{2} \widetilde{V}+\left(3 \widetilde{U} \widetilde{V}_{z}+3 \widetilde{V}^{2}+3 \widetilde{U}^{2} \widetilde{V}+6 c_{0} \widetilde{U} \widetilde{V}\right) & =\mathcal{O}(1 / z)
\end{aligned}
$$

and has a formal expansion given by

$$
\widetilde{U}(z)=\sum_{i=1}^{\infty} \widetilde{u}_{i} z^{-i}, \quad \widetilde{V}(z)=\sum_{i=1}^{\infty} \widetilde{v}_{i} z^{-i}
$$

for constants $\widetilde{u}_{i}$ and $\widetilde{v}_{i}$.

CASE B: Let $(U, V)$ be a solution of (2.4) which is asymptotic to (2.6), and define $\widetilde{U}=U-c_{1}$ and $\widetilde{V}=V+c_{1}^{2}$. Then, $(\widetilde{U}, \widetilde{V})$ solves the system

$$
\begin{aligned}
\widetilde{U}_{z z} & -3 c_{1} \widetilde{U}_{z}+6 c_{1} \widetilde{V}-3 c_{1}^{2} \widetilde{U} \\
& +\left(3 \widetilde{U} \widetilde{U}_{z}+6 \widetilde{U} \widetilde{V}+\widetilde{U}^{3}+3 c_{1} \widetilde{U}^{2}\right)=\mathcal{O}(1 / z) \\
\widetilde{V}_{z z} & +3 c_{1} \widetilde{V}_{z}-3 c_{1}^{2} \widetilde{V}-6 c_{1}^{3} \widetilde{U} \\
& +\left(3 \widetilde{U} \widetilde{V}_{z}+3 \widetilde{V}^{2}+3 \widetilde{U}^{2} \widetilde{V}+6 c_{1} \widetilde{U} \widetilde{V}-3 c_{1}^{2} \widetilde{U}^{2}\right)=\mathcal{O}(1 / z)
\end{aligned}
$$

and has a formal solution given by (3.2).

Following Wasow's theorem, we rewrite each system of two second-order equations (3.1) and (3.3) as a system of four first-order equations

$$
\frac{d Y}{d z}=\mathcal{F}(z, Y), \quad \mathcal{F}(z, Y)=\mathcal{F}_{0}(Y)+\sum_{r=1}^{\infty} \frac{1}{z^{r}} \mathcal{F}_{r}(Y)
$$

where the components of the vector $Y$ are $y_{2 i+1}=\widetilde{U}_{i}$ and $y_{2 i}=\widetilde{V}_{i}$ for $i=0,1$. We then construct the Jacobian of $\mathcal{F}(z, Y)$ evaluated at $Y=0$ as $|z| \rightarrow \infty$,

$$
\mathcal{J}_{A}=\left(\begin{array}{cccc}
0 & 0 & 1 & 0 \\
0 & 0 & 0 & 1 \\
-3 c_{0}^{2} & -6 c_{0} & 3 c_{0} & 0 \\
0 & -3 c_{0}^{2} & 0 & -3 c_{0}
\end{array}\right), \quad \mathcal{J}_{B}=\left(\begin{array}{cccc}
0 & 0 & 1 & 0 \\
0 & 0 & 0 & 1 \\
3 c_{1}^{2} & -6 c_{1} & 3 c_{1} & 0 \\
6 c_{1}^{3} & 3 c_{1}^{2} & 0 & -3 c_{1}
\end{array}\right),
$$

for CASE A and CASE B respectively. Let $\lambda_{A}, \lambda_{B}$ denote the eigenvalues of the matrices $\mathcal{J}_{A}, \mathcal{J}_{B}$. Scaling $\lambda=\lambda_{A} / c_{0}$ and $\lambda=\lambda_{B} / c_{1}$, we obtain

$$
\begin{array}{ll}
\text { Case A : } & \lambda_{1}=\sqrt{3} \exp (i \pi / 6)=-\overline{\lambda_{2}}=-\lambda_{3}=\overline{\lambda_{4}}, \\
\text { CASE B : } & \lambda_{1}=\sqrt{3(5-\sqrt{5}) / 2}=-\lambda_{3}, \quad \lambda_{2}=\sqrt{3(5+\sqrt{5}) / 2}=-\lambda_{4},
\end{array}
$$

where the overbar denotes complex conjugation. Since each eigenvalue is different from zero, all the hypotheses of Wasow's theorem are fulfilled. This proves the existence of true solutions with behaviours that are valid in sectors in the complex $z$ plane with a central angle less than $\pi$. Applying the Boutroux transformation (2.3) gives corresponding sectors of validity in the $x$ plane. These sectors have an angular opening less than $3 \pi / 4$, as given by $\sigma_{A, k, \beta}$ and $\sigma_{B, k}$ in Proposition 3.1 .

We now turn to the second part of Proposition 3.1, which concerns the sectors of validity. These sectors are determined by exponential terms which occur beyond all orders in the asymptotic description of the solutions (see 8 ). Let $\left(U_{0}, V_{0}\right)$ be a 
solution of (2.4) with asymptotic behaviour (2.5) or (2.6) and perturb this solution as follows:

$$
U=U_{0}+\widehat{U}, \quad V=V_{0}+\widehat{V}
$$

where $(\widehat{U}, \widehat{V}) \ll 1$. Substitute (3.7) into equations (2.4). Using the asymptotic behaviour of $\left(U_{0}, V_{0}\right)$, given by (2.5) or (2.6), we find that the perturbation term must satisfy equations (3.1) and (3.3) respectively, with $(\widetilde{U}, \widetilde{V}) \mapsto(\widehat{U}, \widehat{V})$ in each case. Since $(\widehat{U}, \widehat{V}) \ll 1$, the linear terms in the equations are dominant; and hence to determine the asymptotic behaviour of $(\widehat{U}, \widehat{V})$ it is sufficient to consider only these terms:

$$
\begin{aligned}
\widehat{U}_{z z}-3 c_{0} \widehat{U}_{z}+3 c_{0}^{2} \widehat{U}+6 c_{0} \widehat{V} & =\mathcal{O}(1 / z), \\
\widehat{V}_{z z}+3 c_{0} \widehat{V}_{z}+3 c_{0}^{2} \widehat{V} & =\mathcal{O}(1 / z), \\
\widehat{U}_{z z}-3 c_{1} \widehat{U}_{z}+6 c_{1} \widehat{V}-3 c_{1}^{2} \widehat{U} & =\mathcal{O}(1 / z), \\
\widehat{V}_{z z}+3 c_{1} \widehat{V}_{z}-3 c_{1}^{2} \widehat{V}-6 c_{1}^{3} \widehat{U} & =\mathcal{O}(1 / z),
\end{aligned}
$$

for Case A and Case B respectively. Proceeding as above, we write these systems of second-order equations as systems of first-order equations,

$$
\frac{d Y}{d z}=\mathcal{K} Y, \quad \mathcal{K}(z)=\mathcal{J}+\sum_{r=1}^{\infty} \frac{1}{z^{r}} \mathcal{K}_{r},
$$

where $Y$ is a column vector with components $y_{2 i+1}=\widehat{U}_{i}$ and $y_{2 i+2}=\widehat{V}_{i}$, for $i=0,1$, and $\mathcal{K}(z)$ is a matrix of asymptotically expanded coefficients, where $\mathcal{J}$ is defined by $\mathcal{J}_{A}$ or $\mathcal{J}_{B}$ (3.4) in each case.

A fundamental matrix solution of (3.8) is given in [16], Theorem 12.3, by

$$
Y(z)=\mathcal{H}(z) z^{\mathcal{D}} \exp (\mathcal{Q}(z))
$$

where $\mathcal{Q}(z)$ is a diagonal matrix whose elements are linear in $z$ with leading term

$$
z \operatorname{diag}\left(\lambda_{1}, \lambda_{2}, \lambda_{3}, \lambda_{4}\right)
$$

where $\lambda_{i}$ are the distinct eigenvalues of $\mathcal{J}$. The matrix $\mathcal{D}$ is constant diagonal, and the matrix $\mathcal{H}(z)$ has an asymptotic expansion

$$
\mathcal{H}(z) \sim \sum_{r=0}^{\infty} \mathcal{H}_{r} z^{-r}, \quad|z| \rightarrow \infty, \quad \operatorname{det} \mathcal{H}_{0} \neq 0 .
$$

Extracting the asymptotic behaviour of $(\widehat{U}, \widehat{V})$ from $Y$ given by (3.9), we obtain

$$
(\widehat{U}(z), \widehat{V}(z)) \sim \sum_{i=1}^{4} \exp \left(\lambda_{i} z\right) \mathcal{C}_{i},
$$

where $\mathcal{C}_{i}$ is a vector with two arbitrary constant components.

This resultant asymptotic behaviour for $(\widehat{U}, \widehat{V})$ must be consistent with the original assumption that $(\widehat{U}, \widehat{V}) \ll 1$. This is the case only if $\Re\left(\lambda_{i} z\right)<0$ or $\mathcal{C}_{i}=0$, for each $i$ in (3.10). The sectors defined by those $z$ for which $\Re\left(\lambda_{i} z\right)<0$ are

$$
S_{i, l}=\left\{z \in \mathbb{C} \mid a_{i}+2 l \pi<\arg (z)<a_{i}+(2 l+1) \pi\right\}, \quad l \in \mathbb{Z},
$$

where $a_{i}=\pi / 2-\phi_{i}$ and $\phi_{i}=\arg \lambda_{i}$. In each sector $S_{i, l}$ the corresponding constant $\mathcal{C}_{i}$ is free. If two sectors overlap, then in the sector of overlap two constants are 
free. The orientation of the sectors is determined by $a_{i}$, and hence by the eigenvalues (3.5) or (3.6), for CASE A or CASE B. Thus, using the specific values of $a_{i}$ together with the transformation (2.3) we obtain the sectors $\sigma_{A, k, \beta}$ and $\sigma_{B, k}$, and the corresponding number of free parameters of the solutions in these sectors. This concludes the proof of Proposition 3.1

Denote the solutions defined in Proposition 3.1 as follows:

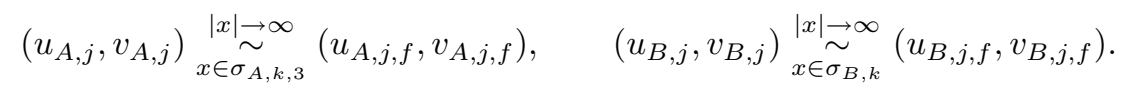

\section{UNIQUe SOLUTIONS}

Proposition 4.1. Given $x_{0} \neq 0$, the true solutions $\left(u_{A, j}, v_{A, j}\right)$ and $\left(u_{B, j}, v_{B, j}\right)$ are unique in the respective sectors

$$
\begin{aligned}
\Sigma_{A, k, j} & =\left\{x \in \mathbb{C}|| x|>| x_{0} \mid, \frac{k \pi}{4}<\arg \left(x-x_{0}\right)<\frac{\left(k+\beta_{k, j}\right) \pi}{4}\right\}, \\
\Sigma_{B, k} & =\left\{x \in \mathbb{C}|| x|>| x_{0} \mid, \frac{(2 k+1) \pi}{8}<\arg \left(x-x_{0}\right)<\frac{(2 k+13) \pi}{8}\right\},
\end{aligned}
$$

where $k=0, \ldots, 7$. For each $k$, $j$ takes two values $j_{1}, j_{2} \in\{1,2,3\}, j_{1} \neq j_{2}$, and $\beta_{k, j_{1}}=4$ and $\beta_{k, j_{2}}=5$.

Remark 4.1. If $j \in\left\{j_{1}, j_{2}\right\}$ for both $k$ and $k+1$, then $\beta_{k, j}=4$ and $\beta_{k+1, j}=5$.

Proof of Proposition 4.1. We prove the result in the transformed $z$ coordinates and then apply (2.3) to obtain the required results in terms of $x$. For some choice of $i$ and $l$, define the following two sectors:

$$
\widehat{S}_{i, l, \pm \epsilon}=\left\{z \in \mathbb{C} \mid a_{i} \pm \epsilon+2 l \pi<\arg z<a_{i} \pm \epsilon+(2 l+1) \pi\right\},
$$

which are related to $S_{i, l}$ (3.11) by rotation by $\pm \epsilon$. Let $\left(U_{1}, V_{1}\right)$ and $\left(U_{2}, V_{2}\right)$ be two solutions whose asymptotic behaviour is known to be (2.5) or (2.6) in the respective sectors $\widehat{S}_{i, l,+\epsilon}$ and $\widehat{S}_{i+1, l,-\epsilon}$. Define the overlap of these two sectors as

$$
\widetilde{S}_{i, l, \epsilon}=\widehat{S}_{i, l,+\epsilon} \cap \widehat{S}_{i+1, l,-\epsilon} .
$$

We have two pieces of information we can exploit. Firstly, the two solutions have the same asymptotic behaviour for $z \in \widetilde{S}_{i, l, \epsilon}$. Thus,

$$
(W, T):=\left(U_{1}-U_{2}, V_{1}-V_{2}\right) \underset{z \in \tilde{\widetilde{S}}_{i, l, \epsilon}}{\stackrel{|z| \rightarrow \infty}{\rightleftharpoons}} o\left(z^{-m}\right),
$$

for all positive integers $m$. Secondly, since $\left(U_{1}, V_{1}\right)$ and $\left(U_{2}, V_{2}\right)$ are both solutions of (2.4), their difference $(W, T)$ must satisfy the system of linear ODEs

$$
\begin{aligned}
W_{z z}-3 U_{1} W_{z} & +\left(-3 U_{2, z}+6 V_{2}+U_{1}^{2}+U_{1} U_{2}+U_{2}^{2}\right) W+6 U_{1} T \\
& =\frac{3}{4 z}\left(\left(U_{1}+U_{2}\right) W-W_{z}\right)+\frac{1}{8 z^{2}} W \\
T_{z z}+U_{2} T_{z} & +\left(3\left(U_{1}+U_{2}\right) V_{1}+V_{1, z}\right) W+\left(3\left(V_{1}+V_{2}\right)+3 U_{2}^{2}\right) T \\
& =-\frac{1}{4 z}\left(6 V_{1} W+6 U_{2} T+5 T_{z}\right)+\frac{1}{8 z^{2}} T .
\end{aligned}
$$

In this system, the asymptotic expansions of the coefficients of $(W, T)$, and derivatives of these terms, are known; both $\left(U_{1}, V_{1}\right)$ and $\left(U_{2}, V_{2}\right)$ are given by (2.5) or (2.6) for CASE A and CASE B respectively. Thus (4.2) is a linear system which 
can be rewritten in the form (3.8), where $\mathcal{J}$ is defined by $\mathcal{J}_{A}$ or $\mathcal{J}_{B}$ (3.4) in each case, and whose solution is given by (3.9). Extracting the asymptotic behaviour of $(W, T)$ from $Y$ given by (3.9) we obtain

$$
(W, T) \sim \sum_{r=1}^{4} \exp \left(\lambda_{r} z\right) \mathcal{C}_{r}, \quad z \in \widetilde{S}_{i, l, \epsilon},
$$

where $\lambda_{r}$ are the eigenvalues of $\mathcal{J}_{A}$ or $\mathcal{J}_{B}$, and $\mathcal{C}_{r}$ are free vector constants. The two expressions for the asymptotic behaviour of $(W, T)$, (4.1) and (4.3), must be consistent. Since $a_{i}=\pi / 2-\phi_{i}$ and $\phi_{i}=\arg \lambda_{i}$, it is clear that for each $r$ in (4.3) there exists some $z$ in $\widetilde{S}_{i, l, \epsilon}$ for which $\Re\left(\lambda_{r} z\right)>0$. Thus (4.1) and (4.3) are consistent only if each $\mathcal{C}_{r}$ in (4.3) is set to zero. This gives $\left(U_{1}, V_{1}\right)=\left(U_{2}, V_{2}\right)$ in $\widetilde{S}_{i, l, \epsilon}$. The sector of validity for the asymptotic behaviour can be analytically continued into the extended sector $S_{i, l} \cup S_{i+1, l}$.

\section{TROnquéE SOLUTiOnS}

The families of solutions defined by Proposition 3.1 are the analogue of Boutroux's tronquée solutions of $\mathrm{P}_{\mathrm{II}}$, while the 24 solutions defined by Proposition 4.1 (16 asymptotic to (2.1), 8 to (2.2) ) are the analogue of the tri-tronquée solutions. The properties of the solutions of JM $\mathrm{P}_{\mathrm{II}}^{(2)}$ are summarised in Table 1. In particular, the number of special rays internal to the sectors of validity are given with a corresponding name suggested, in the spirit of Boutroux's tronquée and tri-tronquée.

TABLE 1. Tronquée Solutions of JM $\mathrm{P}_{\mathrm{II}}^{(2)}$

\begin{tabular}{|c|c|c|c|c|c|c|c|}
\hline & \multicolumn{5}{|c|}{ CASE A } & \multicolumn{2}{|c|}{ Case B } \\
\hline Parameters & 2 & 2 & 1 & 0 & 0 & 2 & 0 \\
\hline Sector Opening & $\pi / 4$ & $\pi / 2$ & $3 \pi / 4$ & $\pi$ & $5 \pi / 4$ & $3 \pi / 4$ & $3 \pi / 2$ \\
\hline Internal Rays & 1 & 3 & 5 & 7 & 9 & 5 & 11 \\
\hline Tronquée Prefix & - & tri- & penta- & hepta- & ennea- & penta- & hendeca- \\
\hline
\end{tabular}

\section{Discussion}

This paper has demonstrated the existence of new types of tronquée solutions of JM $\mathrm{P}_{\mathrm{II}}^{(2)}$, which extend the results obtained by Boutroux for the classical first and second Painlevé equations. Interestingly, we note that while the alternative fourth-order second Painlevé equation $\mathrm{FN} \mathrm{P}_{\mathrm{II}}^{(2)}$ does not admit these new types of tronquée solutions, it does admit another variation: bi-tronquée solutions.

A further qualitative difference between the two fourth-order equations is the form of the asymptotic expansions of the solutions. In contrast to the expansions for JM $\mathrm{P}_{\mathrm{II}}^{(2)}$ given by (2.1) and (2.2), those for $\mathrm{FN} \mathrm{P}_{\mathrm{II}}^{(2)}$ are given in [10] as

$$
y(x)=\left(\frac{x}{6}\right)^{1 / 4}\left(1+\mathcal{O}\left(x^{-5 / 4(1-\epsilon)}\right)\right) \text { or } y(x)=-\frac{2 \alpha}{5 x}\left(1+\mathcal{O}\left(x^{-5 / 4(1-\epsilon)}\right)\right),
$$

$\epsilon>0$. In particular, we note that an asymptotic expansion of the form $y(x) \sim 1 / x$, which is admitted by $\mathrm{P}_{\mathrm{II}}$ and each $\mathrm{FN}_{\mathrm{II}}^{(n)}$ is not valid for either $u(x)$ or $v(x)$ 
in $\mathrm{JM} \mathrm{P}_{\mathrm{II}}^{(2)}$. This expansion does arise in the next member of the Jimbo-Miwa hierarchy, JM $\mathrm{P}_{\mathrm{II}}^{(3)}$ :

$$
\begin{aligned}
-u_{x x x}+2 v_{x x}+4 u u_{x x}+3 u_{x}^{2}-6 v u_{x}-6 u^{2} u_{x}+u^{4}+12 u^{2} v+6 v^{2}+8 g_{4} x & =0, \\
v_{x x x}+4 u v_{x x}+2 v u_{x x}+2 u_{x} v_{x}+6 v v_{x}+6 u^{2} v_{x}+4 u^{3} v+12 u v^{2} & =8 \alpha_{3} .
\end{aligned}
$$

This equation admits three possible algebraic expansions of the form

$$
u(x)=u_{0}\left(1+\mathcal{O}\left(x^{-5 / 4(1-\epsilon)}\right)\right), \quad v(x)=v_{0}\left(1+\mathcal{O}\left(x^{-5 / 4(1-\epsilon)}\right)\right),
$$

with leading-order behaviours given by

$$
\begin{aligned}
u_{0}=\left(-8 g_{4} x\right)^{1 / 4}, & v_{0}=-2 \alpha_{3}\left(-8 g_{4} x\right)^{-3 / 4}, \\
u_{0}=\left(24 g_{4} x / 7\right)^{1 / 4}, & v_{0}=-\frac{1}{3}\left(24 g_{4} x / 7\right)^{1 / 2}, \\
u_{0}=\frac{-\left(2 \alpha_{3}+g_{4}\right)}{\left(4 g_{4} x\right)}, & v_{0}=\left(-4 g_{4} x / 3\right)^{1 / 2} .
\end{aligned}
$$

It is straightforward to show that the asymptotic expansions for $u(x)$ in the system of equations JM $\mathrm{P}_{\mathrm{II}}^{(2 m-1)}$ are comparable to those of $\mathrm{FN} \mathrm{P}_{\mathrm{II}}^{(m)}$, given in [10].

Another interesting result follows from the degeneracy of $u_{0}$ given in (6.3). If $\alpha_{3}=-g_{4} / 2$, then a special solution of the system $\mathrm{JM} \mathrm{P}_{\mathrm{II}}^{(3)}$ is

$$
u(x)=0, \quad \widehat{v}_{x x}=6 \widehat{v}^{2}+2 g_{4} x, \quad \alpha_{3}=-g_{4} / 2,
$$

where $\widehat{v}=-v / 2$, and the equation for $\widehat{v}(x)$ is equivalent to the classical first Painlevé equation. Similar results hold between all the odd $(n \geq 3)$ members of the Jimbo-Miwa hierarchy and the equations of the first Painlevé hierarchy.

\section{REFERENCES}

1. P. Boutroux, Recherches sur les transcendantes de M. Painlevé et l'étude asymptotique des équations différentielles du second ordre, Ann. Sci. École Norm. Sup. (3) 30 (1913), 255-375. MR:1509163

2. — Recherches sur les transcendantes de M. Painlevé et l'étude asymptotique des équations différentielles du second ordre (suite), Ann. Sci. École Norm. Sup. (3) 31 (1914), 99-159. MR1509174

3. B. Dubrovin, T. Grava, and C. Klein, On universality of critical behaviour in the focusing nonlinear Schrödinger equation, elliptic umbilic catastrophe and the tri-tronquée solution to the Painlevé-I equation (2007), arXiv:0704.0501. To appear in J. Nonlinear Sci.

4. Hermann Flaschka and Alan C. Newell, Monodromy- and spectrum-preserving deformations. I, Comm. Math. Phys. 76 (1980), no. 1, 65-116. MR588248 (82g:35103)

5. Pilar R. Gordoa, Nalini Joshi, and Andrew Pickering, On a generalized $2+1$ dispersive water wave hierarchy, Publ. Res. Inst. Math. Sci. 37 (2001), no. 3, 327-347. MR.1855426 (2002j:35292)

6. Second and fourth Painlevé hierarchies and Jimbo-Miwa linear problems, J. Math. Phys. 47 (2006), no. 7, 073504, 16 pp. MR2250304

7. E. L. Ince, Ordinary Differential Equations, Dover Publications, New York, 1944. MR0010757 $(6,65 f)$

8. A. R. Its and A. A. Kapaev, Quasi-linear Stokes phenomenon for the second Painlevé transcendent, Nonlinearity 16 (2003), no. 1, 363-386. MR1950792 (2003i:34200)

9. Michio Jimbo, Tetsuji Miwa, and Kimio Ueno, Monodromy preserving deformation of linear ordinary differential equations with rational coefficients. I. General theory and $\tau$-function, Phys. D 2 (1981), no. 2, 306-352. MR630674 (83k:34010a)

10. N. Joshi and M. Mazzocco, Existence and uniqueness of tri-tronquée solutions of the second Painlevé hierarchy, Nonlinearity 16 (2003), no. 2, 427-439. MR.1958609 (2004a:34172) 
11. Nalini Joshi, The second Painlevé hierarchy and the stationary KdV hierarchy, Publ. Res. Inst. Math. Sci. 40 (2004), no. 3, 1039-1061. MR2074710(2005c:37139)

12. Nalini Joshi and Martin D. Kruskal, The Painlevé connection problem: An asymptotic approach. I, Stud. Appl. Math. 86 (1992), no. 4, 315-376. MR.1160153 (93c:34013)

13. A. V. Kitaev, Caustics in 1+1 integrable systems, J. Math. Phys. 35 (1994), no. 6, 2934-2954. MR.1275481 (95f:35242)

14. Rupert C. Littlewood, Hyperelliptic asymptotics of Painlevé-type equations, Nonlinearity 12 (1999), no. 6, 1629-1641. MR.1726668 (2000k:34139)

15. Gregory Moore, Geometry of the string equations, Comm. Math. Phys. 133 (1990), no. 2, 261-304. MR1090426 (92a:81184)

16. Wolfgang Wasow, Asymptotic expansions for ordinary differential equations, Dover Publications Inc., New York, 1987, reprint of the 1976 edition. MR919406 (88i:34003)

School of Mathematics and Statistics F07, University of Sydney, Sydney, NSW 2006, Australia

E-mail address: nalini@maths.usyd.edu.au

School of Mathematics and Statistics F07, University of Sydney, Sydney, NSW 2006, Australia

E-mail address: teganm@maths.usyd.edu.au 